\title{
MIR326 Pre-miRNA
}

National Cancer Institute

\section{Source}

National Cancer Institute. MIR326 Pre-miRNA. NCI Thesaurus. Code C82819.

MIR326 is an oligoribonucleotide that is encoded by the human MIR326 gene and has a role in the regulation of gene expression. 